\title{
Role of Molecular Pathology in the Treatment of Anaplastic Gliomas and Glioblastomas
}

\author{
Presented by Matthias Holdhoff, MD, PhD
}

\section{Abstract}

The 2016 WHO Classification of Tumors of the Central Nervous System integrated molecular biomarkers into the classifications of malignant gliomas. Several markers now play a key role in our understanding of these tumors and are integral for clinical decision-making; these include codeletion of $1 \mathrm{p}$ and $19 \mathrm{q}$ and IDH1 and IDH2 mutations. The presentation by Matthias Holdhoff, MD, PhD, at the NCCN 23rd Annual Conference discussed the current understanding of anaplastic gliomas (WHO grade III) in the context of the new classification and its implications on clinical practice. The 2018 NCCN Guidelines for Central Nervous System Cancers incorporate molecular and histologic characteristics in the staging and treatment of both low- and high-grade gliomas (WHO grades II-IV).

J Natl Compr Canc Netw 2018;16(5.5):642-645 doi: 10.6004ljnccn.2018.0045

\begin{abstract}
"Molecular pathology in the context of classification of malignant gliomas has been an increasingly interesting, yet somewhat confusing, topic for many years," said Matthias Holdhoff, MD, PhD, Associate Professor of Oncology and Neurosurgery, Johns Hopkins University School of Medicine, and panel member of the NCCN Guidelines for Central Nervous System (CNS) Cancers, in the introduction to his summary of molecular tests that have been implemented for primary brain cancers. "The recently updated 2016 WHO Classification of Tumors of the CNS provided much needed clarification that has greatly improved our understanding of these cancers and has already impacted clinical care," he explained.

At the NCCN 23rd Annual Conference, Dr. Holdhoff presented the historic context of our understanding of gliomas, using a simplified graphic of the previous WHO Classification of gliomas, which divided them into 2 major histopathologic types: oligodendrogliomas and astrocytomas. A third type, oligoastrocytomas, were historically considered "mixed" gliomas. Further, he explained that tumors were defined based on their growth activity as either low-grade (grade II) or high-grade
\end{abstract}

Presented by Matthias Holdhoff, MD, PhD, Sidney Kimmel

Comprehensive Cancer Center at Johns Hopkins, Baltimore, Maryland.

Dr. Holdhoff has disclosed that he has served as a scientific advisor for

AbbVie, Inc. and Celgene Corporation, and that he received support for

travel from Arbor Pharmaceuticals, LLC.

Correspondence: Matthias Holdhoff, MD, PhD, Sidney Kimmel

Comprehensive Cancer Center at Johns Hopkins, 1550 Orleans Street,

1M16, Baltimore, MD 21287. Email: mholdho1@jhmi.edu (grade III and IV) tumors. In 2016, for the first time, the WHO integrated molecular criteria in their classification of gliomas. ${ }^{1}$ There is now a significantly improved understanding of the fundamental biological difference between oligodendrogliomas and astrocytomas, which are now merely separated based on presence or absence of biomarkers that have direct impact on clinical practice.

He further provided a historical synopsis (2005 to present) illustrating the emerging importance of the 3 most widely used molecular biomarkers in gliomas ${ }^{1-13}$ : 06-methylguanine-DNA methyltransferase (MGMT) promoter methylation (confers improved outcome in glioblastoma [GBM] and overall better response to temozolomide), codeletion of $1 \mathrm{p}$ and $19 \mathrm{q}$ (confers improved prognosis in anaplastic oligodendroglioma; patients derive significant benefit from chemotherapy with the PCV regimen [procarbazine, lomustine, and vincristine]), and most recently, IDH1 and IDH2 mutations (associated with lower grade tumors, younger age, and improved outcomes) (Figure 1). "These 3 markers are currently the most clinically relevant markers in managing gliomas," he emphasized.

\section{Codeletion of $1 p$ and $19 q$}

"Codeletion of $1 p$ and $19 q$ has become a key clinical biomarker. It is both prognostic and predictive, and it is considered a 'tie breaker' to determine what is a true oligodendroglioma versus what is, if no codeletion is detected, 


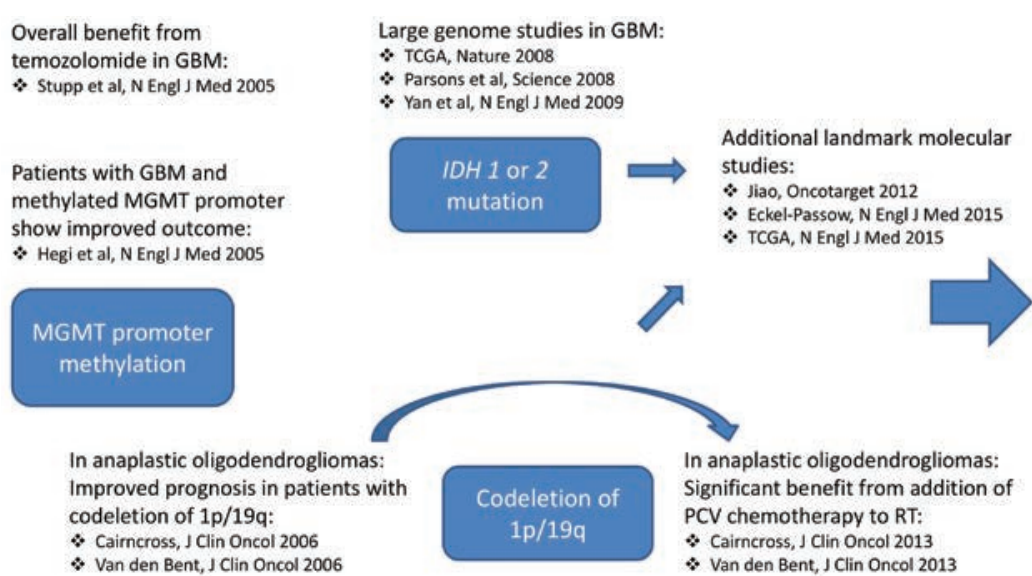

Figure 1. A simplified "evolution" of our understanding of gliomas in the context of key biomarkers.

Abbreviations: GBM, glioblastoma; MGMT, 06-methylguanine-DNA methyltransferase; PCV, procarbazine, lomustine, and vincristine; RT, radiation therapy.

better understood as an astrocytoma," Dr. Holdhoff explained. It is also associated with mutations in IDH, CIC, and FUBP1..$^{14}$

Two randomized prospective trials ${ }^{5,6}$ examined the role of chemotherapy with PCV plus radiation therapy (RT) in patients with anaplastic oligodendroglioma and anaplastic oligoastrocytoma histology. Both studies illustrated the importance of codeletion of $1 \mathrm{p}$ and $19 \mathrm{q}$ as a prognostic and predictive biomarker. RTOG 9402 treated patients with 4 cycles of PCV followed by RT, ${ }^{5}$ and EORTC 26951 evaluated RT followed by 6 cycles of PCV. "The impressive improvement in overall survival with the addition of PCV to RT highlights the biological importance of the codeletion and shows that these tumors are biologically very different from astrocytomas, which lack this marker," Dr. Holdhoff emphasized.

The overall improvement in survival for patients treated with PCV in addition to RT was immense. In RTOG 9402, median overall survival was 14.7 years in codeleted patients in the combination therapy arm versus 7.3 years for those who received RT alone. ${ }^{5}$ Median survival was not reached in the combination arm of EORTC 26951.6 "Results of these studies were astonishing for these otherwise sostubborn gliomas," Dr. Holdhoff said. "These are rare cancers though. If this was breast cancer, it would be all over The New York Times!"

Currently, there are 2 important ongoing prospective trials based on the presence or absence of codeletion of $1 \mathrm{p}$ and $19 \mathrm{q}$. The CODEL study in patients with newly diagnosed $1 \mathrm{p} / 19 \mathrm{q}$ codeleted oligo- dendrogliomas and anaplastic oligodendrogliomas is comparing treatment with RT followed by PCV versus RT plus concomitant temozolomide followed by adjuvant temozolomide (ClinicalTrials.gov identifier: NCT00887146). Temozolomide and PCV have previously been used in conjunction with RT to treat anaplastic oligodendrogliomas, but to date there has not been a head-to-head comparison between the 2 regimens; RT and PCV is currently the most evidencebased treatment.

The CATNON study (ClinicalTrials.gov identifier: NCT00626990) aims to define the role of temozolomide in the treatment of non-codeleted anaplastic gliomas, which, based on the current WHO classification, would be considered (anaplastic) astrocytomas. Interim data from CATNON have been published, and results support a role for adjuvant temozolomide in the therapy for patients with noncodeleted anaplastic gliomas. ${ }^{15}$

\section{IDH1 and IDH2 Mutations}

IDH1 and IDH2 are metabolically active enzymes, Dr. Holdhoff explained. IDH mutations are present in most grade II-III gliomas. ${ }^{10}$ "We still only know parts of the exact biological relevance of these markers. We know a mutated IDH leads to production of an oncometabolite [D2-hydroxyglutarate] that causes epigenetic changes in affected cells," he told listeners.

The new 2018 NCCN Guidelines for CNS Cancers stipulate that IDH mutation testing is required 
for the workup of glioma, with mutation-specific immunohistochemistry as the recommended first test. If negative, then sequencing is recommended in certain clinical situations to detect less common mutations of IDH1 or IDH2. Standard sequencing methods include Sanger sequencing, pyrosequencing, and next-generation sequencing (performed on formalin-fixed paraffin-embedded tissue specimens).

IDH mutations are commonly associated with MGMT promoter methylation and a relatively favorable prognosis; conversely, IDH wild-type is associated with an increased risk of aggressive disease. IDH1/2 mutations are also important for stratification of clinical trials.

\section{MGMT Promoter Methylation}

MGMT is a DNA repair enzyme that reverses the DNA damage caused by alkylating agents such as temozolomide, which results in tumor resistance to temozolomide and nitrosourea-based chemotherapy.

Approximately $45 \%$ of GBMs are methylated. ${ }^{3}$ MGMT promoter methylation status can be detected with methylation-specific PCR, pyrosequencing, or array-based testing. MGMT promoter methylation is associated with IDH mutations and genome-wide epigenetic changes. MGMT promoter methylation has prognostic value, conferring a survival advantage, best described in GBM, and a higher likelihood of benefit from alkylating chemotherapy with temozolomide. "In a nutshell, methylated MGMT status is 'good' feature to have," he emphasized.

However, studies also suggest that unmethylated patients may derive benefit from temozolomide. In the original EORTC-NCIC study that defined the role of temozolomide in the treatment of patients with newly diagnosed GBM, methylated patients had 2-year survival rates of $22.7 \%$ with RT versus $46 \%$ with $\mathrm{RT} /$ temozolomide, and unmethylated patients experienced 2 -year survival rates of $<2 \%$ with RT compared with $13.8 \%$ with RT/temozolomide. ${ }^{2,3}$ Similar findings supporting the benefit from the addition of temozolomide to RT in MGMT unmethylated patients were reported in a more recently published randomized prospective study in older patients with newly diagnosed GBM. ${ }^{16}$ "One can therefore argue that combination therapy with RT and temozolomide is a reasonable option for any patient with newly diagnosed GBM who is thought to be well enough to tolerate this treatment, independent of their MGMT promoter methylation status," Dr. Holdhoff said.

\section{Other Important Markers}

Testing using large genomic panels is becoming more widely used among institutions and can identify other potentially targetable markers, but the overall benefit from genome-wide testing in the care of individual patients with brain cancers has yet to be defined.

"Understandably, many patients are interested in learning as much as possible about their cancer and ask for genomic testing. It is possible that in 5 to 10 years, genome-wide testing will have a more established role in the treatment of these cancers," he commented. Other noteworthy markers that can be detected by genome-wide testing include alterations in ATRX, TERT, and BRAF.

\section{Treatment of Anaplastic Gliomas}

The 2018 NCCN Guidelines recommend multidisciplinary input for treatment planning. Maximal safe resection provides tissue for diagnosis. "If it is determined that the tumor is an anaplastic oligodendroglioma (ie, $1 \mathrm{p} / 19 \mathrm{q}$ codeleted), and the patient has a good performance status (Karnofsky performance status $[\mathrm{KPS}] \geq 60$ ), then treatment with RT and PCV is the most evidence-based treatment to date; per the NCCN Guidelines, it is a category 1 recommendation," he stated.

Newly diagnosed patients with anaplastic astrocytomas without $1 \mathrm{p} / 19 \mathrm{q}$ codeletions are most commonly treated with RT and temozolomide, treatment extrapolated from the data in GBM. The NCCN Guidelines list different available treatment options with their respective supporting level of evidence in the literature.

Patients with anaplastic gliomas and a poor performance status (KPS <60) may require a different approach: fractionated external-beam RT alone or temozolomide (category $2 \mathrm{~B}$ ) or palliative treatment with best supportive care. "Anaplastic gliomas are still considered incurable and tend to grow back at some point in time. Patients require continuous follow-up. Regarding treatment options, especially at time of recurrence, one size does not fit all, and we encourage an ongoing multidisciplinary discussion," he stated. 


\section{References}

1. Louis DN, Perry A, Reifenberger G, et al. The 2016 World Health Organization classification of tumors of the central nervous system: a summary. Acta Neuropathol 2016;131:803-820.

2. Stupp R, Mason WP, van den Bent MJ, et al. Radiotherapy plus concomitant and adjuvant temozolomide for glioblastoma. N Engl J Med 2005;352:987-996.

3. Hegi ME, Diserens AC, Gorlia T, et al. MGMT gene silencing and benefit from temozolomide in glioblastoma. N Engl J Med 2005;352:997-1003.

4. Cairncross G, Berkey B, Shaw E, et al. Phase III trial of chemotherapy plus radiotherapy compared with radiotherapy alone for pure and mixed anaplastic oligodendroglioma: Intergroup Radiation Therapy Oncology Group trial 9402. J Clin Oncol 2006;24:2707-2714.

5. Cairncross G, Wang M, Shaw E, et al. Phase III trial of chemoradiotherapy for anaplastic oligodendroglioma: long-term results of RTOG 9402. J Clin Oncol 2013;31:337-343

6. van den Bent MJ, Brandes AA, Taphoorn MJ, et al. Adjuvant procarbazine, lomustine, and vincristine chemotherapy in newly diagnosed anaplastic oligodendroglioma: long-term follow-up of EORTC brain tumor group study 26951. J Clin Oncol 2013;31:344-350.

7. van den Bent MJ, Carpentier AF, Brandes AA, et al. Adjuvant procarbazine, lomustine, and vincristine improves progression-free survival but not overall survival in newly diagnosed anaplastic oligodendrogliomas and oligoastrocytomas: a randomized European Organisation for Research and Treatment of Cancer phase III trial. J Clin Oncol 2006;24:2715-2722.

8. Parsons DW, Jones S, Zhang X, et al. An integrated genomic analysis of human glioblastoma multiforme. Science 2008;321:1807-1812.
9. Cancer Genome Atlas Research Network. Comprehensive genomic characterization defines human glioblastoma genes and core pathways [published correction appears in Nature 2013;494:506]. Nature 2008;455:1061-1068.

10. Yan H, Parsons DW, Jin G, et al. IDH1 and IDH2 mutations in gliomas. N Engl J Med 2009;360:765-773.

11. Jiao Y, Killela PJ, Reitman ZJ, et al. Frequent ATRX, CIC, FUBP1 and IDH1 mutations refine the classification of malignant gliomas. Oncotarget 2012;3:709-722.

12. Eckel-Passow JE, Lachance DH, Molinaro AM, et al. Glioma groups based on $1 \mathrm{p} / 19 \mathrm{q}, \mathrm{IDH}$, and TERT promoter mutations in tumors. N Engl J Med 2015;372:2499-2508.

13. Cancer Genome Atlas Research Network, Brat DJ, Verhaak RG, et al. Comprehensive, integrative genomic analysis of diffuse lower-grade gliomas. N Engl J Med 2015;372:2481-2498.

14. Bettegowda C, Agrawal N, Jiao Y, et al. Mutations in CIC and FUBP1 contribute to human oligodendroglioma. Science 2011;333:1453-1455.

15. van den Bent $M$, Baumert $B$, Erridge $S$, et al. Interim results from the CATNON trial (EORTC study 26053-22054) of treatment with concurrent and adjuvant temozolomide for $1 \mathrm{p} / 19 \mathrm{q}$ non-co-deleted anaplastic glioma: a phase 3, randomised, open-label intergroup study. Lancet 2017;390:1645-1653.

16. Perry JR, Laperriere N, O'Callaghan CJ, et al. Short-course radiation plus temozolomide in elderly patients with glioblastoma. N Engl J Med 2017;376:1027-1037. 